\title{
Evaluating Transit Market Potential and Selecting Locations of Transit Service Facilities Using GIS
}

\author{
Srinivas S. Pulugurtha and Shashi S. Nambisan, \\ UNLV Transportation Research Center \\ Nanda Srinivasan, Cambridge Systematics Inc.
}

\section{Abstract}

Accessibility to transit service facility (TSF) locations plays a significant role in the success of public transportation systems. The ease with which the end-user can reach a TSF (e.g., bus stops, rail stations, or multimodal centers) plays prominently in the decision-making process of the individual.

This article presents a working definition for transit market potential based on accessibility in terms of walking distance and walking time. Further, a measure is constructed to evaluate transit market potential for TSF locations for a transit system. The measure of transit potential is represented by an index value based on demographic criteria such as employment, household size, vehicle ownership, etc. This index can be used to identify locations of TSFs that increase a route's potential for ridership. A methodology is proposed to estimate the Index of Transit Potential for TSFs. This methodology involves (I) identifying the accessible network of streets around each TSF that is within an acceptable access threshold for a transit rider, and (2) estimating the transit market potential based on key demographic characteristics. The analytical and visualization capabilities of a Geographic Information Systems (GIS) 
program are utilized to help attain the objective. A case study is used to demonstrate the application of the methodology. In the case study, a portion of a route of the Las Vegas Citizens Area Transit (CAT) system is analyzed and the Index of Transit Potential is estimated. The index values are then used to locate TSFs along the route. This is compared with the existing stop locations for the route.

\section{Introduction}

Increasing congestion on roadways is a problem of concern not only to transportation system managers but also to elected officials, public administrators, and the general public. Potential demand management solutions to alleviate congestion include encouraging carpooling, promoting transit usage by providing effective public transportation systems, and reducing vehicle trips in general. The success of any public transit system depends on several factors including service frequency, fares, reliability, TSF locations, accessibility, comfort, convenience, and safety. The spacing and location of TSFs are major determinants of system availability and reliability (Regional Transportation Commission of Clark County [RTC] 1997). To enhance passenger convenience and to ensure desirable operating speeds of buses, transit agencies develop guidelines for spacing stops on a route. For example, the stop spacing identified in guidelines used by the CAT system operating in the Las Vegas, Nevada, metropolitan area ranges from 152 to 213 meters (500 to $700 \mathrm{feet}$ ) in high-density residential locations and 335 to 457 meters $(1,100$ to 1,500 feet $)$ in low-density residential locations. However, such guidelines are based on general rules of thumb. This article presents a methodology to support developing such guidelines and to locate TSFs.

\section{Background}

The primary criteria that influence the locations of TSF include accessibility to potential passengers, safety, transit route configuration (grid or radial network, express or local route, etc.), and traffic operations. Consistency in TSF locations, especially for transit systems that share the right-of-way with other vehicles, minimizes confusion for potential patrons, transit operators, and for other vehicles. Typically, nearside, farside, and midblock locations are possible 
for stops on transit facilities. In the Las Vegas metropolitan area, bus stops are normally located at the farside of an intersection approach since most of the stops are on through travel lanes (RTC 1997). This decision was made in order to minimize delays to other vehicles at the intersection because of signal-timing considerations. Other advantages of farside stop locations for a bus transit system include the following (RTC 1997):

- Passengers boarding and alighting are less likely to cross in front of the bus.

- Reduced interference with traffic at intersections where there are heavier traffic volumes on the approach than on the departure leg.

- Stopped buses do not obstruct sight lines to the left for vehicles entering the intersection from a side street.

- Sight distance is improved for pedestrians.

Another general criterion includes avoidance of proximity to driveway or alleyways.

Typical transit systems have three main types of TSFs: transfer stops, time-check stops, and other general stops. In practice, sites of transfer stops are decided based on the network configuration. Time-check stops are TSFs where transit vehicles stop regardless of whether there are passengers to board or alight. The intent of time-check stops is generally to facilitate schedule adherence (to the extent possible) along sections between such stops. The general stops are TSFs at which the transit vehicles stop only if there are boarding or alighting passengers. This article addresses the location of the general stops or general TSFs.

In order to facilitate time-coordinated transfers for passengers between various routes, it would be advantageous to establish a transfer TSF also as a time-check TSF. Then, if the schedules of routes that traverse this site are coordinated, it would minimize the delays to passengers transferring between these routes. There could be a minimum of 4 transit routes operating at a site that is the intersection of 2 two-way streets (or rail lines). The spatial and temporal distributions of the transit passenger flow would influence the decision to coordinate none, some, or all of the routes at this site. The decision to locate a TSF 
at a transfer point should consider the walking distance, walking time, and the transit schedule (scheduled departure time). Such transfer and time-check TSFs typically account for a very small portion of all the designated stops along a bus route. For example, there are 6 intermediate time-check stops that also are transfer stops along Route 101 of the CAT system. This route has a total of 28 stops in the southbound direction (i.e., transfer and time-check stops account only for about 21 percent of all the stops). Thus, on a typical transit system, the majority of the TSFs are those termed "general stops." They need to be located based on factors and considerations that are in addition to schedule coordination issues.

\section{Review of the Literature}

A recent analysis based on the 1995 Nationwide Personal Transportation Survey (NPTS) showed that transit's share in urban areas larger than 3 million persons is 3.77 percent, while for urban areas with 0.5 million to 1 million persons it averages 0.88 percent (Chu 1998). However, transit's share of trips by persons with annual household incomes less than $\$ 15,000$ or living within one block of a bus stop are 11.75 percent and 7.96 percent, respectively, for areas larger than 3 million persons, and 2.19 percent and 2.26 percent, respectively, for areas with 0.5 million to 1 million persons. These statistics reflect the need to approximately locate TSFs if the objective is to increase the ridership. The focus of this article is to identify the best (or good) locations of TSFs based on accessibility and transit market potential.

Accessibility, or access opportunity, is defined as "the spatial quality of the relation between location of infrastructure facilities and the location of the users" (Bach 1981). Thus, it is the ease with which TSFs (say, bus stops) can be reached by a given population set. Distance and time are two measures of accessibility. A facility is considered accessible to the user if the accessibility measure is less than an acceptable, predefined maximum value.

Henk and Hubbard (1996) state that transit service coverage refers to the spatial proximity of transit service throughout an urban area. They evaluated potential by including a variety of transit system characteristics measured by 
urban area demographic characteristics such as population, urbanized land area, and population density. Bach (1981) concluded that the type of distance measure and the level of aggregation influence the indices of accessibility and access opportunity.

GIS has traditionally been used in analysis, postprocessing of results, and visual representation of data to facilitate easier recognition of spatial correlation between data and allow for easier decision-making (Environmental Systems Research Institute, Inc. 1997). O’Neill et al. (1992) described a procedure for performing service area analysis on transit routes using a GIS software program. A route's accessibility was indicated by the total number of persons living within the service area. Gomez and Zhao (1998) presented a GISbased methodology that improves the estimate of pedestrian access to transit by utilizing street network information, land-use data, and household information from census data.

Johnston (1966) studied important aspects of transit service and settlement patterns by constructing an index of accessibility. Henk and Hubbard (1996) state that the index of accessibility should be a measure based on various transit system characteristics. Evaluation of accessibility indices for transportation networks have been presented by Sathisan and Srinivasan (1998) and Srinivasan and Sathisan (1998). However, no attempt to integrate accessibility, market potential, and the location of TSFs has been documented in the literature.

\section{Objective}

The methodology presented here is intended to help select locations of TSFs based on an evaluation of transit market potential along a route. It aims to automate the procedure using GIS software. The methodology is based on establishing thresholds for the spatial extent of the market potential along a route, and quantifying the market potential at various locations of TSFs along the route. The spatial extent of the potential market is quantified based on walking distance and walking time considerations.

The analytical capabilities of ARC/INFO, a GIS program, have been employed to assess and analyze spatial data such as those for Traffic Analysis Zone (TAZ) including population by income and age groups, physically hand- 
icapped groups, etc. The data used for the case study were those available from the 1990 census.' An algorithm is proposed for this purpose. Steps 1 and 2 of this algorithm require a network analysis that is available in several popular GIS software packages (e.g., ARC/INFO, TransCad) used in transportation planning. Step 3 needs an overlay of data layers, a primary capability of any GIS software package. Once an index is defined in Step 4, a GIS package can be further used in determining the best locations and routes. For illustration purposes, a section of Route 101 of the CAT system is considered. This section traverses predominantly residential neighborhoods. The methodology presented aims at maximizing the potential transit ridership (i.e., the transit market potential).

In this article, walking time is used to measure accessibility. The first step would be to estimate the total population that has access to a TSF. Not all the population in the accessible region are likely to use the transit system. So, criteria have to be developed to estimate transit market potential for locating a TSF, specifically those that are not transfer/time-check points on a route.

\section{Problem Definition}

Consider a hypothetical TSF along a route in the transit network. The possibility that a resident in that locality will use the facility depends on several factors. These factors include age and gender of the person, income level, automobile ownership, employment, trip purpose, travel distance, physical mobility constraints, walking time (or distance) from the residence to the TSF, temporal considerations (e.g., time of the day, day of the week), access to other transport modes, and relative costs for using each mode.

The walking time to the TSF for the user is a measure of accessibility to the TSF. A potential user will not utilize the transit system if the walking time is very high (i.e., there is an upper limit to this walking time, whereas the lower limit can be as low as feasible, maybe a few seconds). For illustration purposes, consider five minutes to be the upper limit for the walking time (this may be changed depending on the actual situation). The walking time for two individuals traversing the same distance may be different (i.e., walking time is stochastic in nature). For simplicity, it is assumed that the walking speed is the 
same for all individuals, and it is about 1.2 meters per second ( 4 feet per second). The maximum walking distance for a user is the product of the threshold for walking time and the walking speed. Thus, for the case at hand, the maximum walking distance for a user will be equal to approximately 366 meters (1,200 feet). This is roughly 0.25 mile, which is a generally accepted premise of most transit operators. The accessible zone for this TSF is the area within the specified upper limit or threshold of walking time (or distance) from the TSF.

The creation of an accessible zone around the TSF is discussed later in this article. The zone is now defined to be accessible to all the residents in it. But, not all residents are likely to use the transit system. So, certain criteria have to be defined in order to identify the transit market potential. Using the estimated transit market potential for various TSF locations along a route, the objective is to find an optimal set of TSFs that maximize the transit market potential.

\section{Index of Transit Potential}

Transit-demand ridership depends on fare and other dummy variables (e.g., strikes) in addition to the factors identified in the previous section (McLeod et al. 1991). Neglecting fare and the dummy factors, the following demographic variables are significant in evaluating transit market potential:

- age group (less than 18 years and greater than 55 years)

- household income (less than $\$ 15,000$ per year)

- household size (greater than 3)

- household auto ownership (less than or equal to 1 )

- all unemployed persons

- all physically handicapped persons

These variables are presented solely for illustrative purposes. They may be changed or redefined, and other variables may be added as appropriate.

An index is constructed based on the potential captive riders for each of the variables presented above. This index, the Index of Transit Potential, is a measure of the transit market potential for each TSF. The ratio of the number of users based on a variable (one of the six defined above) who are within the 
accessible zone to the total value of the same variable in the study region multiplied by 1,000 (for normalization purposes) is first determined. The Index of Transit Potential is obtained by summing the ratios for each of the six variables described above. This can be mathematically expressed as shown in Equation 1 .

Index of Transit Potential $j=\Sigma_{i} \quad\left(\frac{n_{i j}}{N_{i}} \times 1000\right)$

where:

$j$ is the TSF number.

$i$ denotes categories such as unemployed, age, household size, vehicle ownership, physically handicapped, etc.

$n_{i j}$ is the number of users belonging to category $i$ in the region served by a TSF $j$.

$N_{i}$ is the total number of users belonging to category $i$ in the study region.

The value for each variable is defined to a scale of 1,000 . This value of 1,000 does not affect the TSF ranking (used in the selection process), and hence the selection process. All the variables are weighted equally, but, if warranted, individual weights can be assigned to each variable.

\section{Methodology}

Estimating the Index of Transit Potential means identifying the number of potential captive riders in the accessible zone. This requires spatial analysis that can be carried out using a GIS program. The following five steps are used in the process.

\section{Step 1: Simulate TSF Locations along a Route}

The street network of the study area is used as a starting point. This is represented as a "line" coverage in the GIS environment. Transit routes are also represented on this network. Along a desired route, TSF locations are simulated with a fixed distance between two adjacent TSFs. The TSFs are coded as a "point" coverage in the GIS environment. Once the TSF locations are simulated, the next step is to find the boundaries for the accessible zones. 


\section{Step 2: Define the Accessible Zone around a TSF}

In the second step, the capabilities of the GIS program are used to create a buffer around each simulated TSF. As an example, consider the previous discussion which noted that a user would at most walk for about 366 meters (1,200 feet). Using the "allocate" feature of the GIS program, a subnetwork (or allocated network) is created along the links near the TSF, joining all the points on the links that are at a distance of no more than 366 meters around each TSF. Thus, the walking distance from any point in this subnetwork to the TSF is either less than or equal to 366 meters. There are three possible ways of creating the accessible zone for measuring the accessibility to TSFs.

Method 1: Arc Lengths of Accessible Arcs Method. In the Arc Length method, it is assumed that the demographic criterion (or one of the six variables of interest to quantify transit market potential) is distributed proportionally to the lengths of the streets that are present in each TAZ. This assumes that the population distribution is proportional to the distribution of the street network within the TAZ - which is reasonable. The steps involved in delineating the demographic characteristics are:

1. Overlay the demographic coverage (TAZ) on the allocated coverage.

2. Estimate the lengths of "accessible" arcs for each TSF location that are present in each unit of the demographic coverage (TAZ).

3. Find the ratio of the accessible arc lengths to the total lengths of all arcs for each TAZ. This ratio is defined as the "arc ratio" for the TAZ. Thus, a percentage of the base unit of the demographic coverage is allocated for each TSF. This can be used for subsequent isolation of demographic data.

An illustration of a TSF location, the street network and TAZs near it, and portions of the links (arcs) on the street network that meet the criterion for accessibility (i.e., walking distance from the TSF is less than 366 meters) is shown in Figure 1(a). The Arc Length method has inherent advantages as it is relatively easy and eminently suited for implementation in a GIS environment. The disadvantage is that a particular segment of a route falling on a TAZ boundary is allocated to one of the two TAZs and not to both. 


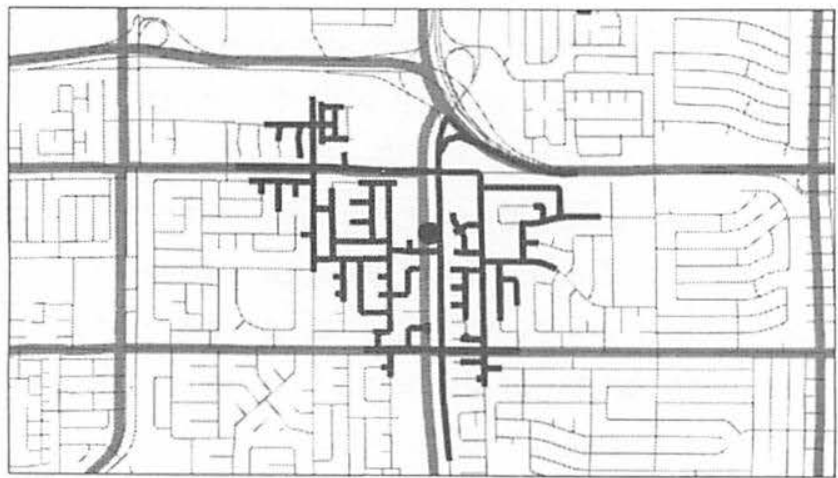

Legend

(a)

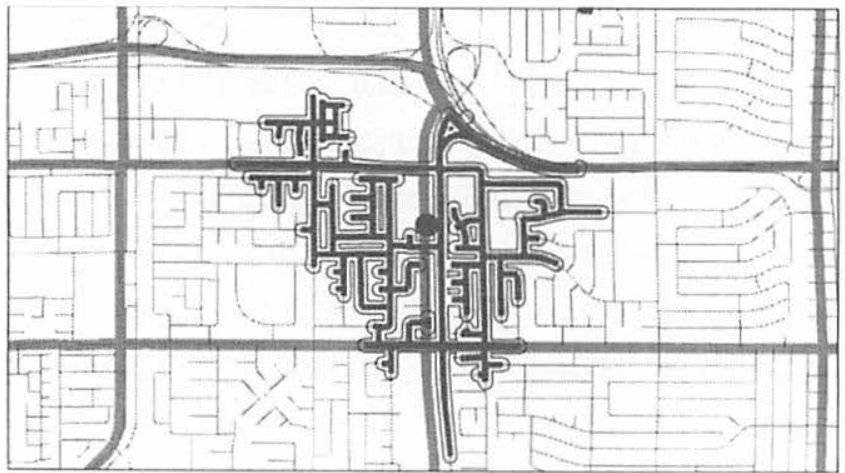

Buffer around accessible arcs

(b)

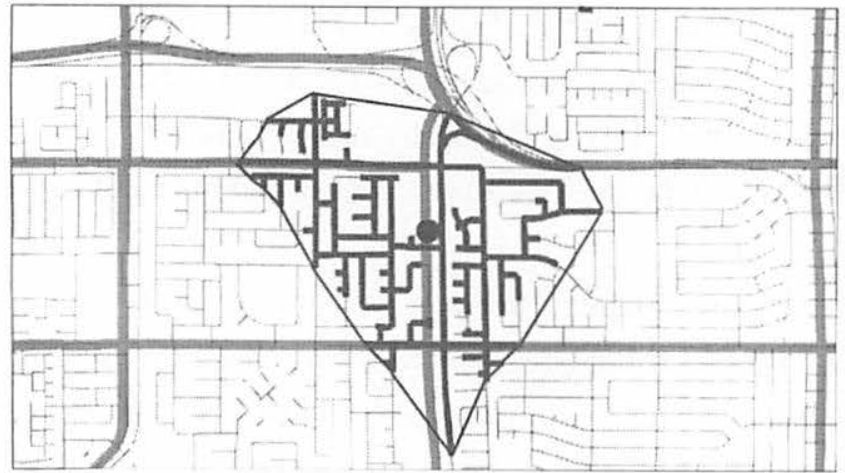

Hull polygon encompassing end points of accessible arcs

(c)

Figure 1. Regions around TSFs for measuring transit market potential 
Method 2: Buffer Around Accessible Arcs Method. The second method is to create a small buffer along the allocated arcs as shown in Figure 1(b). This assumes that the demographic variables are uniformly distributed throughout the TAZ. The steps involved in estimating data for each of the six variables of interest are:

1. Overlay the TAZ coverage on the allocated buffers.

2. Estimate the proportion of TAZs by area allocated to each TSF. This ratio is defined as the "buffer ratio."

3. Use this proportion to subsequently quantify data for each of the six demographic variables of interest.

This method solves the disadvantage of the first method, and thus helps correct the allocation of arcs to the TAZs. However, this approach raises other questions. For example, what if there was a huge apartment complex along the link (it cannot be divided into parts, as normally it is presumed that the TSF is accessible to the whole complex), or what if one of the arcs is identified as a freeway or the freeway passes through the polygon? These problems outweigh the benefits of solving the disadvantage of the Arc Length of Accessible Arcs method.

Method 3: Hull Polygon Method. The third method is a hull-type buffer shown in Figure 1(c). This is obtained by joining the farthest points of the accessible arcs as a polygon. The following steps are involved:

1. Overlay the TAZ coverage and identify the TAZs to which this polygon belongs.

2. Assuming that the demographic characteristics are uniformly distributed over these TAZs, the ratio of the area within the hull polygon to the total TAZ area is estimated and defined to be the "hull area ratio."

3. Estimates for each of the six demographic variables for each TAZ are evaluated as the product of the hull area ratio and the value of that variable for the TAZ. If the hull polygon encompasses more than one TAZ, the respective contribution of each TAZ is added to obtain the value for the TSF.)

But there are certain limitations as in the previous case, such as what if one of the arcs identified is a freeway, or if a freeway passes through the polygon. 
Any of the above approaches essentially involves a spatial overlay, which is easy to visualize in a GIS-based environment. The three types of spatial overlays can be performed using one of these data coverages:

- Overlay on land parcel coverage (obtained from the assessor's office).

- Overlay on census block data developed by the Census Bureau.

- Overlay on the TAZ coverage developed by the local metropolitan planning organization (i.e., the RTC in the Las Vegas metropolitan area).

The estimated measures reflect the level of accessibility of the simulated TSF to a population set. So, the final results obtained will reflect the level of detail or approximation used. The second and third types of overlay provide significant details required for the methodology. But, the street network coverage does not exactly mesh with the second and third types of coverage as the RTC developed these coverages from a different base source. Hence, due to the nature of available data, only the Arc Length of Accessible Arcs method is evaluated.

\section{Step 3: Estimate the Index of Transit Potential for Each TSF}

Consider the variables identified in the Index of Transit Potential section. Using the Census Transportation Planning Package (CTPP) report (U.S. DOT, FHWA 1995) developed for the TAZs, the number of users corresponding to each of the selected variables is estimated for each TAZ. From the proportion obtained in Step 2, the number of potential captive riders of each variable is allocated to each TSF. An illustration of this step is shown in Table 1. The first column in the table represents the TSF number, the second column is for TAZs for which this TSF is the closest, and the arc ratio for each TAZ is shown in the third column. Values for each of the six variables for the TAZ are shown in the next six columns; the last six columns show the contributions from each TAZ for each of the six variables (this is the product of the value of the variable for the TAZ and the arc ratio for the TAZ). The Index of Transit Potential for each TSF is calculated using Equation 1.

\section{Step 4: Locate Candidate TSFs}

The objective of this step is to locate the TSF locations along various routes. It is easy to simulate a large number of TSFs and select the optimal set 


\section{Table 1}

\section{Estimating Transit Market Potential Using Arc Lengths for Accessible Arcs Method}

\begin{tabular}{|c|c|c|c|c|c|c|c|c|c|c|c|c|c|c|}
\hline \multirow[t]{2}{*}{ TSF } & \multirow{2}{*}{$\begin{array}{c}\text { Contributing } \\
\text { TAZ }\end{array}$} & \multirow[t]{2}{*}{ Ratio } & \multicolumn{6}{|c|}{ Variable Value for $\operatorname{TAZ}^{\mathrm{a}}$} & \multicolumn{6}{|c|}{ Variable Value for TSF ${ }^{a}$} \\
\hline & & & 1 & 2 & 3 & 4 & 5 & 6 & 1 & 2 & 3 & 4 & 5 & 6 \\
\hline \multirow[t]{4}{*}{83} & 409 & 0.18 & 22 & 4 & 28 & 1 & 13 & 14 & 3.92 & 0.71 & 5.02 & 0.22 & 2.35 & 2.44 \\
\hline & 410 & 0.07 & 11 & 5 & 58 & 25 & 27 & 53 & 0.77 & 0.34 & 4.08 & 1.77 & 1.88 & 3.74 \\
\hline & 423 & 0.29 & 17 & 8 & 10 & 19 & 22 & 5 & 4.94 & 2.19 & 2.89 & 5.55 & 6.43 & 1.45 \\
\hline & 424 & 0.12 & 59 & 37 & 16 & 31 & 25 & 26 & 7.06 & 4.46 & 1.95 & 3.67 & 2.97 & 3.12 \\
\hline \multicolumn{3}{|c|}{ Sum } & 109 & 54 & 112 & 76 & 87 & 98 & 16.69 & 7.70 & 13.94 & 11.21 & 13.63 & 10.75 \\
\hline \multirow[t]{3}{*}{90} & 443 & 0.08 & 28 & 22 & 49 & 32 & $\overline{55}$ & 63 & 0.08 & 2.21 & 1.76 & 3.95 & 2.57 & 4.38 \\
\hline & 444 & 0.22 & 6 & 5 & 7 & 8 & 6 & 2 & 0.22 & 1.42 & 1.02 & 1.46 & 1.66 & 1.22 \\
\hline & \multicolumn{2}{|l|}{ Sum } & 34 & 27 & 56 & 40 & 60 & 65 & 3.63 & 2.78 & 5.41 & 4.23 & 5.60 & 5.46 \\
\hline
\end{tabular}

a. 1,2,3,4,5, and 6 represent age, income, unemployment, household size, physical mobility, and automobile ownership, respectively.

of TSFs for each route, based on the ranking given by the index value. A section of Route 101 of the CAT system is used to illustrate the procedure. Route 101 is about 20.7 kilometers $(68,000$ feet) long. The section selected is about 11 kilometers ( 36,000 feet) long and has 23 existing TSFs. But, instead of simulating 23 TSFs, 90 locations are simulated with a spacing of about 122 meters (400 feet). The 122-meter (400-foot) spacing is fixed, assuming that there will not be a significant difference in the ridership number if TSFs are located anywhere in between. In practice, based on the route conditions, spacing between TSFs can be made as small as necessary. Using the procedure discussed earlier, accessible zones are created for each of the simulated TSFs. For each simulated TSF, the potential captive riders based on the six variables and the Index of Transit Potential for each TSF are estimated. All the simulated TSFs are sorted based on the index values. Of these, locations with the highest index values are selected (subject to a minimum spacing between adjacent TSF locations) for providing the maximum accessible transit system along the route.

\section{Step 5: Select TSF Locations}

Since the methodology identifies the location of all the simulated TSFs, the spacing can be automatically determined. But, this depends on the number of TSFs selected along a route. A set of criteria is proposed for selecting the number of TSF locations and the spacing: 
1. Select all TSF locations with an index value greater than the mean index value for the route.

2. TSFs with ranks better or equal to the minimum number of TSFs required are selected.

3. The spacing between TSFs affects walking time as well as onboard riding time along a route, thus influencing transit demand.

None of these criteria consider spacing between TSFs. It can be logically interpreted that locating TSFs with very small spacing provides maximum accessibility. But, this is not economically nor operationally feasible. This stresses the need for solving the problem considering both accessibility and spacing between TSFs. This can be done by adding a constraint restricting spacing between two consecutive facilities.

\section{Case Study}

A section of Route 101 of the Las Vegas metropolitan area local transit system is considered for the case study. This section is 36,000 feet long. As discussed in Step 4, 90 TSF locations are simulated along this route with a fixed distance of about 122 meters ( 400 feet) between two consecutive TSFs. Points at a distance of about 366 meters are identified around each TSF. A typical street network that provides accessibility to a TSF is shown in Figure 1(a). This subnetwork, along with the street network, is overlayed on the TAZ coverage. The ratio of lengths of accessible arcs in each TAZ to the total lengths of all arcs lying in the TAZ is estimated for each TSF. The number of potential captive riders based on the selected demographic variables and Index of Transit Potential are estimated for each TSF.

The average index value obtained for the section (considering all 90 simulated stops) is 0.59 . If all the locations with index values greater than 0.59 were selected, then 17 TSF locations need to be provided. Since the maximum walking distance is set at 366 meters, the maximum spacing between two TSFs under ideal conditions is 731.5 meters (2,400 feet). A user located exactly between two TSFs has to walk a maximum distance.

Twenty-three TSF locations with the highest index values were selected along the route to provide exactly the same number of TSFs as exist currently. 
The accessibility region for the 23 selected TSF locations is shown in Figure 2 . Some stretches of the route have large spacing between stops. This simply reflects the spatial distribution of the variables used in the objective function.

The TSF identity (stop number), index values for each variable (age, income, unemployment, household size, mobility, and vehicle), and Index of Transit Potential for the existing and selected 23 locations are shown in Table 2. The locations are arranged in descending order based on the transit poten-

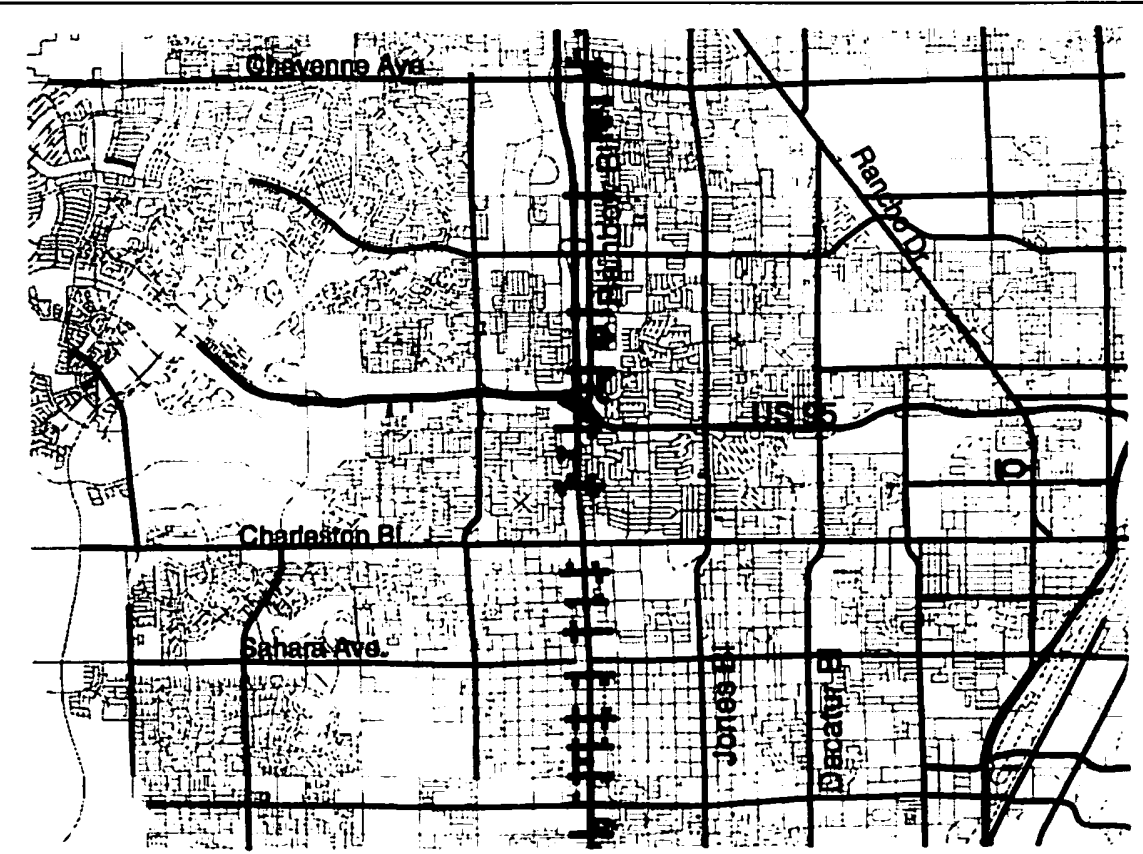

\section{Legend}

$\checkmark$ Major streets

Street network

Access to selected TSFs

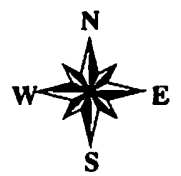

Figure 2. Accessible network for the selected TSF locations 
tial. The average index value for each variable and the Index of Transit Potential of the selected locations is greater than that of the existing locations. The highest Index of Transit Potential for an existing stop is observed as 11.48, whereas the highest Index of Transit Potential for a selected (or simulated) stop is 8.50. This is because the simulated stop does not include the existing stop with the highest Index of Transit Potential. This can be taken care of by simulating bus stops with small spacing.

\section{Discussion}

The results obtained from this study are different from those obtained from the procedure used by the local transit authorities. This may be due to the following:

- The case study uses only data related to residential locations for selecting the TSFs. But, other vital land-use aspects such as commercial centers and offices were not accounted for in the analysis. However, they can easily be incorporated as TAZ attributes.

- Socioeconomic and political aspects were not addressed.

- The approach does not consider data at microscopic levels. Thus, finally it might result in locating the TSF exactly in the middle of an intersection. This may be an optimal location from a market potential point of view, but it may not be feasible for practical reasons. The normal procedure in the Las Vegas area is to opt for farside locations at the intersection. But, this may result in a different index value for the TSF.

- Another aspect ignored in the study is route spacing. "The route spacing and route length though have unique values over time, will have a significant affect on the passenger access time," state Chang and Schonfeld (1995). This problem can be taken care of by simultaneously considering all the routes, with all TSF locations in the transit system.

The case study demonstrates that the methodology presented is a useful decision-support tool for transit system operators. Transit demand depends on the spatial distribution of potential users along a route. Hence, the spacing between TSFs should vary along a route. The methodology presented selects TSF locations based on the Index of Transit Potential. However, various other 
Table 2

Index Values for Each Category and Accessibility Index for TSF Locations

\begin{tabular}{|c|c|c|c|c|c|c|c|}
\hline $\begin{array}{l}\text { Stop } \\
\text { No. }\end{array}$ & $\begin{array}{c}\text { Age } \\
\text { Index }\end{array}$ & $\begin{array}{l}\text { Income } \\
\text { Index }\end{array}$ & $\begin{array}{c}\text { Unemployment } \\
\text { Index }\end{array}$ & $\begin{array}{c}\mathrm{HH} \\
\text { Index }\end{array}$ & $\begin{array}{l}\text { Mobility } \\
\text { Index }\end{array}$ & $\begin{array}{l}\text { Vehicle } \\
\text { Index }\end{array}$ & $\begin{array}{l}\text { Access } \\
\text { Index }\end{array}$ \\
\hline \multicolumn{8}{|c|}{ Existing Stops } \\
\hline 23 & 1.46 & 2.11 & 2.79 & 1.73 & 2.08 & 1.32 & 11.48 \\
\hline 10 & 0.62 & 1.05 & 0.88 & 0.75 & 0.90 & 0.61 & 4.82 \\
\hline 11 & 0.88 & 0.63 & 0.55 & 0.72 & 0.57 & 1.04 & 4.38 \\
\hline 12 & 0.69 & 0.40 & 0.42 & 0.67 & 0.43 & 0.80 & 3.42 \\
\hline 13 & 0.38 & 0.21 & 0.19 & 0.25 & 0.21 & 0.44 & 1.68 \\
\hline 12 & 0.42 & 0.16 & 0.15 & 0.24 & 0.19 & 0.49 & 1.64 \\
\hline 9 & 0.25 & 0.14 & 0.21 & 0.36 & 0.17 & 0.32 & 1.46 \\
\hline 1 & 0.18 & 0.22 & 0.17 & 0.14 & 0.13 & 0.21 & 1.05 \\
\hline 17 & 0.31 & 0.14 & 0.13 & 0.08 & 0.19 & 0.12 & 0.97 \\
\hline 3 & 0.21 & 0.08 & 0.09 & 0.17 & 0.08 & 0.29 & 0.91 \\
\hline 2 & 0.19 & 0.09 & 0.09 & 0.15 & 0.08 & 0.26 & 0.84 \\
\hline 16 & 0.26 & 0.12 & 0.11 & 0.06 & 0.16 & 0.10 & 0.81 \\
\hline 20 & 0.13 & 0.14 & 0.10 & 0.18 & 0.10 & 0.13 & 0.77 \\
\hline 21 & 0.13 & 0.14 & 0.10 & 0.17 & 0.10 & 0.13 & 0.77 \\
\hline 4 & 0.15 & 0.06 & 0.06 & 0.12 & 0.06 & 0.22 & 0.67 \\
\hline 18 & 0.19 & 0.09 & 0.09 & 0.06 & 0.12 & 0.07 & 0.63 \\
\hline 19 & 0.14 & 0.10 & 0.09 & 0.10 & 0.10 & 0.09 & 0.61 \\
\hline 5 & 0.10 & 0.08 & 0.05 & 0.08 & 0.06 & 0.12 & 0.49 \\
\hline 15 & 0.10 & 0.07 & 0.06 & 0.05 & 0.07 & 0.04 & 0.39 \\
\hline 14 & 0.02 & 0.02 & 0.02 & 0.02 & 0.02 & 0.01 & 0.12 \\
\hline 7 & 0.01 & 0.02 & 0.01 & 0.01 & 0.01 & 0.01 & 0.08 \\
\hline 6 & 0.00 & 0.00 & 0.00 & 0.00 & 0.00 & 0.01 & 0.02 \\
\hline 8 & 0.00 & 0.00 & 0.00 & 0.00 & 0.00 & 0.00 & 0.00 \\
\hline Average & 0.30 & 0.26 & 0.28 & 0.27 & 0.25 & $0,3.0$ & 1.65 \\
\hline \multicolumn{8}{|c|}{ Selected Stops } \\
\hline 89 & 0.90 & 2.01 & 1.04 & 0.83 & 1.51 & 2.21 & 8.50 \\
\hline 87 & 0.69 & 0.58 & 0.83 & 0.63 & 0.83 & 1.01 & 4.57 \\
\hline 90 & 0.53 & 0.95 & 0.53 & 0.40 & 0.93 & 1.10 & 4.45 \\
\hline 40 & 0.49 & 1.00 & 0.52 & 0.44 & 0.82 & 0.76 & 4.03 \\
\hline 41 & 0.65 & 0.49 & 0.64 & 0.78 & 0.42 & 0.43 & 3.42 \\
\hline 36 & 0.42 & 0.63 & 0.51 & 0.43 & 0.57 & 0.56 & 3.12 \\
\hline 84 & 0.45 & 0.25 & 0.43 & 0.53 & 0.28 & 0.28 & 2.22 \\
\hline 38 & 0.19 & 0.63 & 0.20 & 0.13 & 0.46 & 0.42 & 2.03 \\
\hline 43 & 0.40 & 0.25 & 0.28 & 0.47 & 0.24 & 0.23 & 1.87 \\
\hline 50 & 0.36 & 0.22 & 0.24 & 0.42 & 0.22 & 0.20 & 1.64 \\
\hline 33 & 0.27 & 0.15 & 0.42 & 0.36 & 0.16 & 0.23 & 1.60 \\
\hline 88 & 0.12 & 0.21 & 0.17 & 0.09 & 0.22 & 0.31 & 1.12 \\
\hline 7 & 0.24 & 0.09 & 0.19 & 0.34 & 0.09 & 0.10 & 1.06 \\
\hline 32 & 0.14 & 0.08 & 0.23 & 0.19 & 0.08 & 0.12 & 0.85 \\
\hline 46 & 0.20 & 0.05 & 0.10 & 0.23 & 0.07 & 0.05 & 0.70 \\
\hline 29 & 0.13 & 0.07 & 0.15 & 0.14 & 0.10 & 0.11 & 0.70 \\
\hline 60 & 0.20 & 0.09 & 0.05 & 0.08 & 0.12 & 0.08 & 0.61 \\
\hline 42 & 0.10 & 0.07 & 0.10 & 0.12 & 0.06 & 0.06 & 0.51 \\
\hline 64 & 0.15 & 0.07 & 0.04 & 0.06 & 0.10 & 0.06 & 0.48 \\
\hline 1 & 0.05 & 0.16 & 0.04 & 0.03 & 0.08 & 0.10 & 0.46 \\
\hline 16 & 0.08 & 0.08 & 0.07 & 0.10 & 0.06 & 0.05 & 0.45 \\
\hline 5 & 0.10 & 0.04 & 0.08 & 0.14 & 0.04 & 0.04 & 0.43 \\
\hline 77 & 0.07 & 0.07 & 0.10 & 0.07 & 0.05 & 0.06 & 0.42 \\
\hline Average & 0,30 & 0.36 & 0,30 & 0.31 & 0,33 & 0.37 & 1.97 \\
\hline
\end{tabular}


aspects related to operations should also be considered in the final solution. This research opens up avenues to examine ways of estimating the transitdemand origin matrix based on land-use patterns; demographic characteristics of the region; and demand variation over time of day, month, and year. Further, the functional classification of roads should be incorporated in the analysis so as to eliminate freeways and other facilities (that do not permit pedestrian or bicycle traffic) from the accessible network.

\section{Conclusions}

The objective of this study was to define a measure for accessibility to each TSF and use the measure to identify optimal locations for TSFs. An Index of Transit Potential, a measure for accessibility, is defined based on the potential captive riders belonging to various demographic variables. A methodology to identify locations for TSFs along various routes was proposed and its use was demonstrated. It is best suited to locate general TSFs (i.e., those that are not time-check points or transfer points). The need for spatial analysis shows the increasing emphasis in solving problems easily using GIS-based environments. The methodology presented serves as a good decision-support tool for designing and operating transit systems. Thus, the final decisions on TSF locations should also consider other factors from an operations point of view.

\section{Acknowledgments}

The authors thank the staff at the RTC of Clark County, Nevada, for providing data and information that were essential for this work. Further, they thank two anonymous referees for their valuable comments.

They also acknowledge a grant from TRW Environmental Safety Systems, Inc., which provided partial support for this work.

\section{Endnote}

1. The Las Vegas metropolitan area has seen a tremendous amount of change in population in the last decade; however, this does not have any bearing on the proposed methodology. 


\section{References}

Bach, L. 1981. The problem of aggregation and distance for analysis of accessibility and access opportunity in location allocation models. Environment and Planning $A$ 13: 955-978.

Chang, S. K., and P. M. Schonfeld. 1995. Optimal dimensions of bus service zones. ASCE Journal of Transportation Engineering 119(4): 567-585.

Chu, Xuehao. 1998. 1995 NPTS analyzed to develop information base on public transit. CUTRlines 9(3): 1-6.

Environmental Systems Research Institute, Inc. 1997. Understanding GIS-The ARC/INFO method. New York: John Wiley Sons, Inc.

Gomez, A., and F. Zhao. April 1998. Improving transit access estimate in transportation modeling using GIS. Paper presented at the 11 th annual GIS for Transportation Symposium. Salt Lake City, Utah.

Henk, R. H., and S. M. Hubbard. 1996. Developing an index of transit service availability. Transportation Research Record 1521: 12-19.

Johnston, R. J. 1966. An index accessibility and its use in the study of bus services and settlement patterns. Journal of Economic and Social Geography 57: 33-38.

McLeod Jr., M. S., K. J. Flannelly, L. Flannelly, and R. W. Behnke. 1991. A multivariate, time series model of transit ridership based on historical aggregate data: The past, present, and future of Honolulu. Paper submitted for 70th annual meeting of the Transportation Research Board. Washington, DC.

O’Neill, W. A., D. R. Ramsey, and J. Chou. 1992. Analysis of transit service areas using geographic information systems. Transportation Research Record 1364: 131-138.

Regional Transportation Commission of Clark County. 1997. Citizens Area Transit: Bus stop guidelines. Las Vegas, NV: Planning Division.

Sathisan, S. K., and Nanda Srinivasan. 1998. Evaluation of accessibility of urban transportation networks. Transportation Research Record 1617: 78-83.

Srinivasan, N., and S. K. Sathisan. May 1998. Quantification and analysis of transportation system accessibility. Proceedings of the Transportation, Land-Use and Air Quality: Making the Connection Conference, Sponsored by the American Society of Civil Engineers. Portland, Oregon, 208-217.

U.S. Department of Transportation, Federal Highway Administration. 1995. CTPP handbook: An instructional guide to the 1990 Census Transportation Planning Package, FHWA-PD-95-019. 


\section{About the Authors}

SRINIVAS S. Pulugurtha (pss@trc.unlv.edu) is an assistant research professor at the Transportation Research Center, University of Nevada, Las Vegas. He received his Ph.D. in civil engineering (focus in transportation) from the University of Nevada, Las Vegas in 1998.

Shashi S. Nambisan (shashi@ce.unlv.edu) is a professor of civil engineering and director of the Transportation Research Center at the University of Nevada, Las Vegas.

NANDA SRINIVASAN (n_18Ø@yahoo.com) is a GIS programmer for Cambridge Systematics. He earned his M.S.E. from the University of Nevada, Las Vegas in 1997. His current efforts include work on the Census Transportation Planning Package for 2000. 